\title{
A Distinct Contribution of the Frontal Eye Field to the Visual Representation of Saccadic Targets
}

\author{
Behrad Noudoost, ${ }^{1}$ Kelsey L. Clark, ${ }^{1}$ and Tirin Moore ${ }^{1,2}$ \\ ${ }^{1}$ Department of Neurobiology, Stanford University School of Medicine, Stanford, California 94305, and ${ }^{2}$ Howard Hughes Medical Institute, Stanford, \\ California 94305
}

\begin{abstract}
The responses of neurons within posterior visual cortex are enhanced when response field (RF) stimuli are targeted with saccadic eye movements. Although the motor-related activity within oculomotor structures seems a likely source of the enhancement, the origin of the modulation is unknown. We tested the role of the frontal eye field (FEF) in driving presaccadic modulation in area $\mathrm{V} 4 \mathrm{by}$ inactivating FEF neurons at retinotopically corresponding sites within the macaque monkey (Macaca mulatta) brain. As previously observed, FEF inactivation produced profound, and spatially specific, deficits in memory-guided saccades, and increased the latency, scatter, and duration of visually guided saccades. Despite the clear behavioral deficits, we found that rather than being eliminated or reduced by FEF inactivation, presaccadic enhancement of V4 activity was increased. FEF inactivation nonetheless diminished the stimulus discriminability of V4 visual responses both during fixation and in the presaccadic period. Thus, without input from the FEF, V4 neurons signaled more about the direction of saccades and less about the features of the saccadic target. In addition, FEF inactivation significantly increased the suppressive effects of non-RF stimuli on V4 activity. These results reveal multiple sources of presaccadic modulation in V4 and suggest that the FEF contributes uniquely to the presaccadic specification of visual target features.
\end{abstract}

Key words: extrastriate cortex; eye movements; motor command; prefrontal cortex; top-down control; visual attention

\section{Introduction}

We shift our gaze to an object of interest $\sim 150,000$ times each day. It is known that the selection of visual targets for saccadic eye movements is accompanied by perceptual benefits for stimuli near the goal of impending saccades (Deubel and Schneider, 1996). Simultaneous with this perceptual benefit is an enhancement in the representation of the target throughout the visual system. Originally observed in the superior colliculus (SC), many neurons exhibit enhanced visual responses when response field (RF) stimuli are selected as saccadic targets (Mohler and Wurtz, 1976). A similar presaccadic enhancement is also observed in other saccade-related areas such as the frontal eye field (FEF) and the lateral intraparietal area (LIP) even among neurons without pure motor properties (Wurtz and Mohler, 1976; Colby et al., 1996). Within posterior visual cortex, presaccadic enhancement of visual responses has been reported for neurons within inferotemporal (IT) cortex (Chelazzi et al., 1993), area MT (Buracas and Albright, 2009), and particularly within area V4 (Fischer and Boch, 1981a; Moore et al., 1998). In V4, presaccadic enhancement of visual activity is accompanied by an increase in orienta-

\footnotetext{
Received Sept. 6, 2013; revised Jan. 15, 2014; accepted Jan. 24, 2014.

Author contributions: B.N., K.L.C., and T.M. designed research;B.N., K.L.C., and T.M. performed research; B.N. and K.L.C. analyzed data; B.N., K.L.C., and T.M. wrote the paper.

This work was supported by NIH EY014924, NSF IOB-0546891, an International Brain Research Organization Fellowship to B.N., and HHMI to T.M. We thank D.S. Aldrich and N.A. Steinmetz for technical assistance. The authors declare no competing financial interests.

Correspondence should be addressed to Dr. Behrad Noudoost, Department of Cell Biology and Neuroscience, Room 01 Lewis Hall, Montana State University, Bozeman, MT 59717. E-mail: bnoudoost@montana.edu.

DOI:10.1523/JNEUROSCI.3824-13.2014

Copyright $\odot 2014$ the authors $\quad 0270-6474 / 14 / 343687-12 \$ 15.00 / 0$
}

tion discriminability (Moore and Chang, 2009) and the magnitude of presaccadic activity predicts the visual guidance of corresponding movements (Moore, 1999). Collectively, the above evidence suggests that the preparation of visually guided saccades normally involves a corresponding specification of the target's visual properties within visual cortex.

Although it is reasonable to hypothesize that presaccadic modulation within posterior visual cortex originates from motor-related activity within oculomotor structures, this hypothesis has not been tested. Among the most likely of these candidate sources is the $\mathrm{FEF}$, as it is known that neurons in this area modulate signals within visual cortex during stable gaze (Moore and Armstrong, 2003; Noudoost and Moore, 2011a). FEF neurons show a mix of visual and motor response properties (Bruce and Goldberg, 1985), and their activity reflects the location of covert attention (Armstrong et al., 2009). FEF neurons are reciprocally connected with multiple extrastriate visual areas, including V4 (Stanton et al., 1995), where FEF projects predominantly excitatory inputs directly onto pyramidal neurons (Anderson et al., 2011). To test the contribution of the FEF to presaccadic modulation in visual cortex, we measured the effects of FEF inactivation on V4 activity during visually guided saccades. We found that rather than being eliminated or reduced by FEF inactivation, presaccadic enhancement in V4 increased substantially. However, in addition to increasing surround suppression, FEF inactivation decreased stimulus discriminability, both during fixation and in the presaccadic period. Thus, the inactivation of the FEF exerted opposing effects on presaccadic enhancement and presaccadic discriminability. These results demonstrate that the FEF contributes uniquely to the presaccadic specification of visual tar- 
get features, and reveal multiple sources of presaccadic modulation in V4.

\section{Materials and Methods}

General and surgical procedures. Three male rhesus monkeys (Macaca mulatta; Monkeys HM, SP, BN) were used in these experiments. Monkeys BN and HM were used for the memory-guided saccade experiments. V4 neuronal responses and visually guided behavior were studied before and after FEF inactivation in Monkeys BN and SP (24 and 9 V4 neurons, respectively). All experimental procedures were in accordance with $\mathrm{Na}$ tional Institutes of Health Guide for the Care and Use of Laboratory Animals, the Society for Neuroscience Guidelines and Policies, and Stanford University Animal Care and Use Committee. Each animal was surgically implanted with a head post, a scleral eye coil, and two recording chambers. Two craniotomies were performed on each animal, allowing access to dorsal V4, on the prelunate gyrus, and FEF, on the anterior bank of the arcuate sulcus. Eye position monitoring was performed via the scleral search coil and was digitized at $500 \mathrm{~Hz}$ (CNC Engineering). Eye monitoring, stimulus presentation, data acquisition, and behavioral monitoring were controlled by the CORTEX system. Visual stimuli presented to V4 RFs were $1.2-1.9^{\circ} \times 0.2-0.4^{\circ}$ bar stimuli appearing at four possible orientations $\left(0,45,90\right.$, and $\left.135^{\circ}\right)$. All stimuli were presented on a $29^{\circ} \times$ $39^{\circ}\left(22^{\prime \prime}\right)$ colorimetrically calibrated CRT monitor (Mitsubishi Diamond Pro 2070SB-BK) with medium short persistence phosphors (refresh rate $77 \mathrm{~Hz})$

Single-neuron recording in V4. Single-neuron recordings in awake monkeys were made through a surgically implanted cylindrical titanium chamber (20 mm diameter) overlaying the prelunate gyrus. Electrodes were lowered into the cortex using a hydraulic microdrive (Narashige). Activity was recorded extracellularly with varnish-coated tungsten microelectrodes (FHC) of $0.2-1.0 \mathrm{M} \Omega$ impedance (measured at $1 \mathrm{kHz}$ ). Extracellular waveforms were digitized and classified as single neurons using both template matching and window-discrimination techniques (FHC, Plexon). The RFs of V4 neurons were mapped during a fixation task in which oriented bars were swept across the display to identify the area of visual space from which the recorded neurons responded; RF boundaries were marked by hand based on the audible discharge of V4 neurons in response to the visual stimulus (Hubel and Wiesel, 1968; Haenny and Schiller, 1988). Bar stimuli evoking the largest response were defined as the "preferred" orientation and orthogonal orientations were defined as the "nonpreferred."

Recording, microstimulation, and inactivation of the FEF. Electrophysiological recordings, electrical microstimulation and pharmacological inactivation of the FEF were each performed using a custom-made microinjectrode (Noudoost and Moore, 2011b). Electrical microstimulation with the microinjectrode consisted of a $100 \mathrm{~ms}$ train of biphasic current pulses $(0.25 \mathrm{~ms}$ each phase, $200 \mathrm{~Hz})$ delivered with a Grass stimulator (S88) and two Grass stimulation isolation units (PSIU-6). Current amplitude was measured via the voltage drop across a $1 \mathrm{k} \Omega$ resistor in series with the return lead of the current source. All stimulation was delivered via varnish-coated tungsten microelectrodes of $0.2-1.0 \mathrm{M} \Omega$ impedance (measured at $1 \mathrm{kHz}$ ) contained within the microinjectrode. In each monkey, the FEF was first localized on the basis of its surrounding physiological and anatomical landmarks and the ability to evoke fixedvector saccadic eye movements with stimulation at currents of $<50 \mu \mathrm{A}$.

Using the custom-designed microinjection system, we infused $0.5-1.0$ $\mu \mathrm{l}$ of muscimol at sites within the FEF over a period of 10-15 min. Our system consisted of a 32 -gauge ( $236 \mu \mathrm{m}$ outer diameter) stainless steel cannula containing a $75 \mu \mathrm{m}$, commercially available epoxy-coated, tungsten microelectrode (FHC). The microelectrode was held in place inside the cannula via a cilux T-junction. The electrode was passed through the center of the T-junction and through a PEEK ferrule where it was soldered to a connector for recording. The cannula was attached to a different opening of the T-junction via another ferrule. The drug line, composed of $363 \mu \mathrm{m}$ (outer diameter) polyimide-coated glass tubing (Polymicro Technologies), was attached through a ferrule to the final $\mathrm{T}$-junction opening. The polyimide tubing was then connected to a manual injection drive (Stoelting) and a gas-tight microsyringe (Hamilton) via a series of high precision fluidic valves attached to a fluidic "circuit" board. The T-junctions, ferrules, fluid valves, and fluidic circuit board were all obtained from LabSmith. Because the inner diameters of the tubing and the cannula were equal $(150 \mu \mathrm{m})$, drug flow was steady with a minimum of clogging or hysteresis. To measure fluid flow into the brain, we drew up into the fluid path an oil-dye-oil marker, whose movement inside the polyimide tubing could be observed with the naked eye. The oil in the marker was of low viscosity $(\sim 1$ centistokes) and also served to separate the water-soluble drug from the water-soluble dye (a nontoxic food coloring). Within the $150 \mu \mathrm{m}$ tubing, a $1 \mathrm{~cm}$ movement of the marker indicated a $\sim 170 \mathrm{nl}$ movement of the drug out of the cannula. Because we could measure movements of the dye marker by as little as 1 $\mathrm{mm}$, the volume resolution of the microinfusion system was $<17 \mathrm{nl}$. The inclusion of the tungsten microelectrode within the center of the drug cannula further allowed us to record the activity of single neurons near the center of the delivered drug volume using conventional recording and filtering techniques. Moreover, we could also use standard electrical microstimulation to confirm at each drug site that saccades could be elicited with low currents, and thus that each microinfusion site was within the FEF. The microelectrode typically extended beyond the beveled tip of the cannula by $50-500 \mu \mathrm{m}$. To keep the microelectrode from being damaged when inserting the cannula into the brain, the ferrule connecting it to the T-junction could be rotated three turns counterclockwise, thereby retracting the microelectrode $\sim 1 \mathrm{~mm}$ back into the cannula. Once the cannula was well within the brain, the ferrule could be slowly rotated clockwise and tightened thus positioning the electrode at a known distance beyond the cannula opening. To suppress FEF neuronal activity, we used muscimol, a potent and selective GABAa agonist that has been widely used in studies involving in vivo inactivation of local neuronal activity, particularly in behaving monkey studies (Sommer and Tehovnik, 1997; Dias and Segraves, 1999; Wardak et al., 2002). Similar to previous studies, muscimol was dissolved in physiological saline at a concentration of $5 \mathrm{mg} / \mathrm{ml}$ ( $\mathrm{pH}, 6.5-7.0)$. Before delivery of the drug, the entire fluid delivery system was soaked and thoroughly flushed with cold sterilant (chlorhexidine diacetate, Nolvasan), flushed with sterile water and then allowed to dry. Based on pilot experiments on the time course of the inactivation effects, behavioral testing and electrophysiological recordings began at least $45 \mathrm{~min}$ after completion of muscimol infusion; the effects of muscimol infusion lasted for at least $4 \mathrm{~h}$ (and potentially up to $24 \mathrm{~h}$ ) following infusion, with all data collection being completed well within the $4 \mathrm{~h}$ window. To ensure that there were no lingering drug effects from the previous experimental session, inactivation experiments were always separated from one another by at least $48 \mathrm{~h}$, although in previous studies $24 \mathrm{~h}$ has been sufficient for the oculomotor effects of muscimol injection into the FEF to wear off (Sommer and Tehovnik, 1997; Dias and Segraves, 1999). After 48 h, no lingering effects of the previous muscimol infusion were observed, and no permanent deficits developed over the series of experiments. Saline infusion sessions were interspersed with inactivation experiments.

Behavioral effects of muscimol infusion in FEF. The effects of muscimol infusion in FEF on visually guided saccade behavior are shown in Figure 1. As shown for a sample experiment in Figure $1 A$, FEF inactivation increased the scatter of the landing points of saccades, especially for saccades toward the area of space represented at the infusion site (hereafter referred to as the overlapping location). The saccade scatter (in units of degrees visual angle, dva) was defined as the average distance between pairs of saccade landing points quantified according to the following formula

Saccade Scatter $=\left(2 / n^{\star}(n-1)\right)^{\star} \sum_{i=1}^{n-1} \sum_{j=i+1}^{n} \sqrt{\left(X_{i}-X_{j}\right)^{2}+\left(Y_{i}-Y_{j}\right)^{2}}$.

Where $X$ and $Y$ are the coordinates (in dva) of the landing points of $n$ number of saccades. The average scatter was $0.72 \pm 0.42 \mathrm{dva}$ for saccades toward the overlapping location before inactivation; after FEF inactivation, the average scatter increased to $1.02 \pm 0.65 \mathrm{dva}\left(p<10^{-3}\right)$. For this particular experiment the scatter also increased for saccades to the nonoverlapping location (away condition) within the same hemifield (before $=0.39 \pm 0.23 \mathrm{dva}$; after $=0.56 \pm 0.32 \mathrm{dva}, p<10^{-3}$; note that the scatter values are smaller for the away conditions, even before muscimol infusion, because the target in the away conditions was a small circular 
A
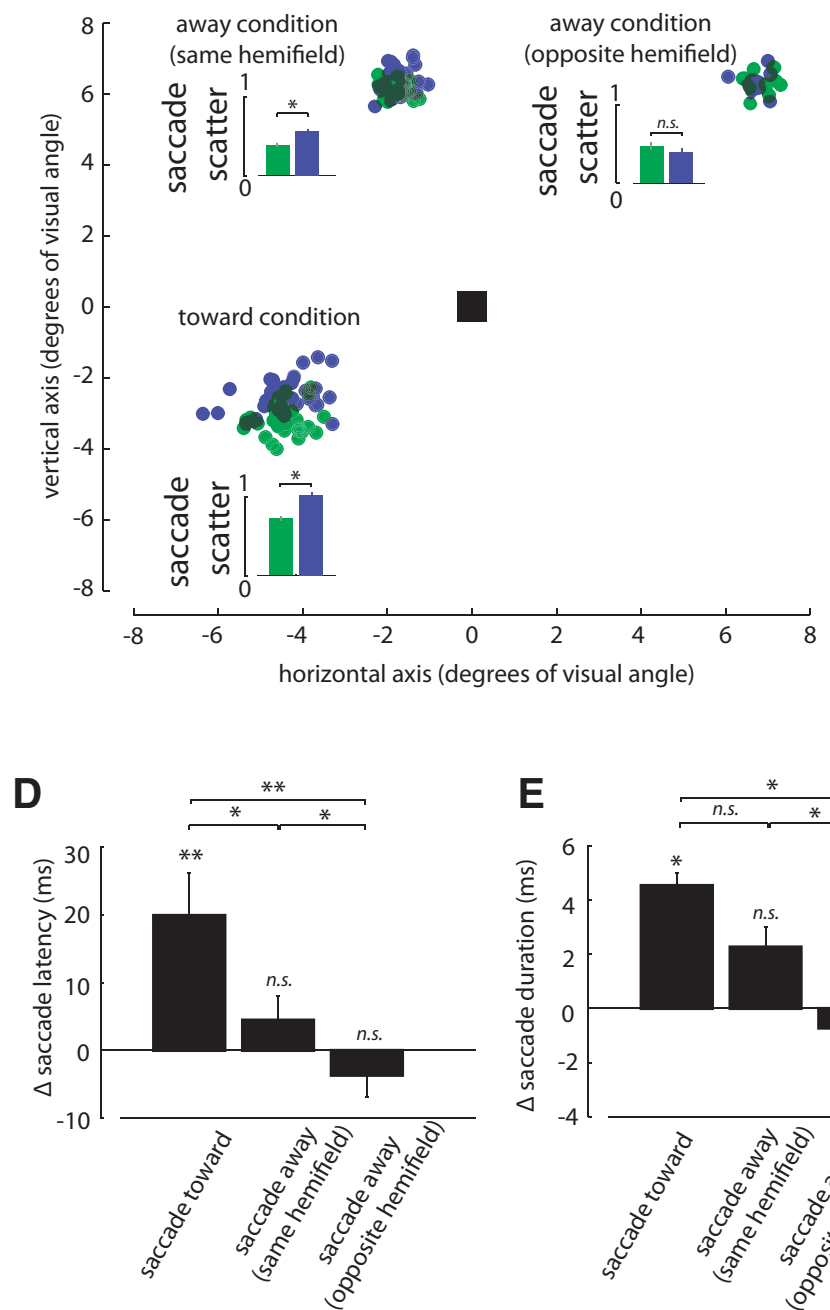

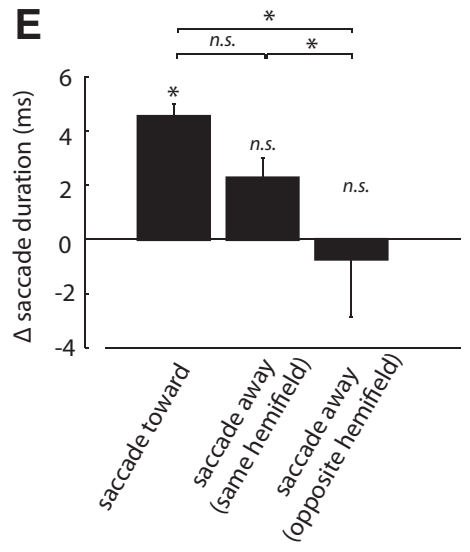

B
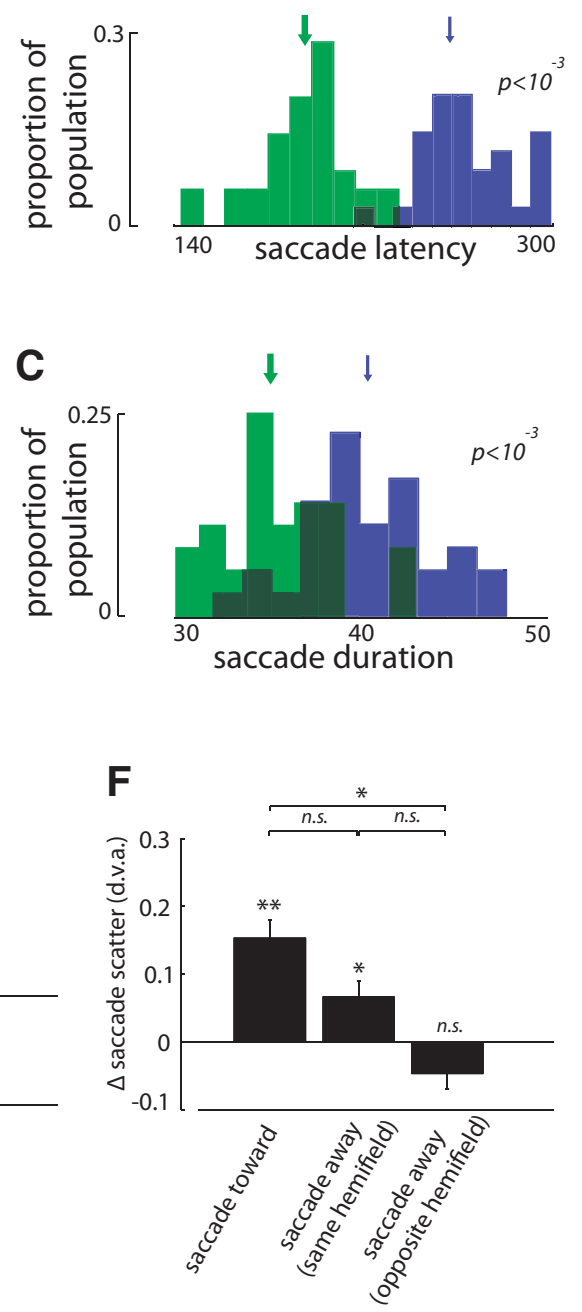

Figure 1. Effects of FEF inactivation on visually guided saccades. $A$, Landing points of saccades in the toward and away conditions before (green) and after FEF inactivation (blue) for a sample experiment. Note that the saccade target is an oriented bar in the toward condition and a circular target in the away conditions. Small bar plots show the mean \pm SE of saccade scatter for each condition. $\boldsymbol{B}, \boldsymbol{C}$, Distributions of saccade latencies and durations before and after inactivation for the sample experiment in $\boldsymbol{A}$. The arrows indicate the median of each distribution. $\boldsymbol{D}-\boldsymbol{F}, \mathrm{Mean}$ and $\mathrm{SEM}$ of changes in latency, duration and scatter of saccades after FEF inactivation in toward and away conditions across 19 experimental sessions. ${ }^{*} p<0.05 ;{ }^{* *} p<0.01 ; n . s .=p>0.05$.

dot rather than the oriented bar presented as the saccade target in the toward condition. The scatter was not significantly different for the away condition when the target appeared in the opposite hemifield (before = $0.47 \pm 0.29 \mathrm{dva}$; after $=0.38 \pm 0.31 \mathrm{dva} ; p=0.085)$. The latency of saccades toward the affected part of space was significantly longer after inactivation (Fig. $1 B$; before $=195 \pm 20 \mathrm{~ms}$, after $=264 \pm 19 \mathrm{~ms}, p<$ $\left.10^{-3}\right)$. However, the reaction time in the away conditions was not affected by FEF inactivation, either for saccades to the same or the opposite hemifield (same hemifield, before $=170 \pm 9 \mathrm{~ms}$ after $=176 \pm 13 \mathrm{~ms}$; $p=0.107$; opposite hemifield, before $=18 \pm 15 \mathrm{~ms}$, after $=179 \pm 10 \mathrm{~ms}$; $p=0.116$ ). The same pattern was observed for saccade duration (Fig. $1 C)$. Saccade duration increased significantly following inactivation only for the toward condition (toward: before $=35 \pm 3 \mathrm{~ms}$, after $=41 \pm 4 \mathrm{~ms}$, $p<10^{-3}$; away-same hemifield: before $=44 \pm 7 \mathrm{~ms}$, after $=47 \pm 7 \mathrm{~ms}$, $p=0.150$; away-opposite hemifield: before $=38 \pm 12 \mathrm{~ms}$, after $=45 \pm$ $17 \mathrm{~ms}, p=0.115)$.

Across 19 experimental sessions of FEF inactivation, the same pattern of increased latency, duration, and scatter for saccades toward the RF is observed. The latency of saccades was affected only in the toward condition (toward condition: before $=213 \pm 18 \mathrm{~ms}$, after $=233 \pm 28 \mathrm{~ms}, p=$ 0.024; away-same hemifield: before $=189 \pm 31 \mathrm{~ms}$, after $=196 \pm 33 \mathrm{~ms}$, $p=0.114$; away-opposite hemifield: before $=192 \pm 22 \mathrm{~ms}$, after $=$ $189 \pm 25 \mathrm{~ms}, p=0.126$; Fig. $1 D$ ). Saccade duration increased signifi- cantly for saccades toward the affected part of space or to targets within the same hemifield, but not for saccades to the opposite hemifield (toward: before $=35 \pm 6 \mathrm{~ms}$, after $=40 \pm 5 \mathrm{~ms}, p<10^{-3}$; away-same hemifield: before $=41 \pm 6 \mathrm{~ms}$, after $=43 \pm 5 \mathrm{~ms}, p=0.033$; awayopposite hemifield: before $=43 \pm 11 \mathrm{~ms}$, after $=42 \pm 8 \mathrm{~ms}, p=0.901$; Fig. $1 E$ ). Average scatter for the toward condition was $0.75 \pm 0.12^{\circ}$ before inactivation, which increased to $0.90 \pm 0.20^{\circ}\left(p<10^{-3}\right.$; Fig. $\left.1 F\right)$. There was also a trend toward increased scatter in the away condition when the target stimulus appeared within the same hemifield as the inactivation site (before $=0.47 \pm 0.20^{\circ}$, after $=0.54 \pm 0.19^{\circ}, p=0.052$ ). Endpoint scatter was not significantly different before versus after inactivation for saccades to targets in the opposite hemifield (before $=0.49 \pm 0.12^{\circ}$, after $=0.44 \pm 0.13^{\circ}, p=0.147$ )

We also measured the monkeys' ability to remember the location of targets across variable delays in a staircase-based, memory-guided saccade task (Fig. 2A). In the memory guided saccade task, a cue appears briefly in one of several locations: the monkey must remember the cue location throughout a variable delay and make saccade to the remembered location upon the removal of the fixation point. The delay duration was adjusted via a staircase paradigm, in which the delay for each trial was incremented up or down based on performance in the previous trial at that location; a correct trial resulted in a juice reward and an increase in the delay at that location, and incorrect trial gave no reward and a de- 
A

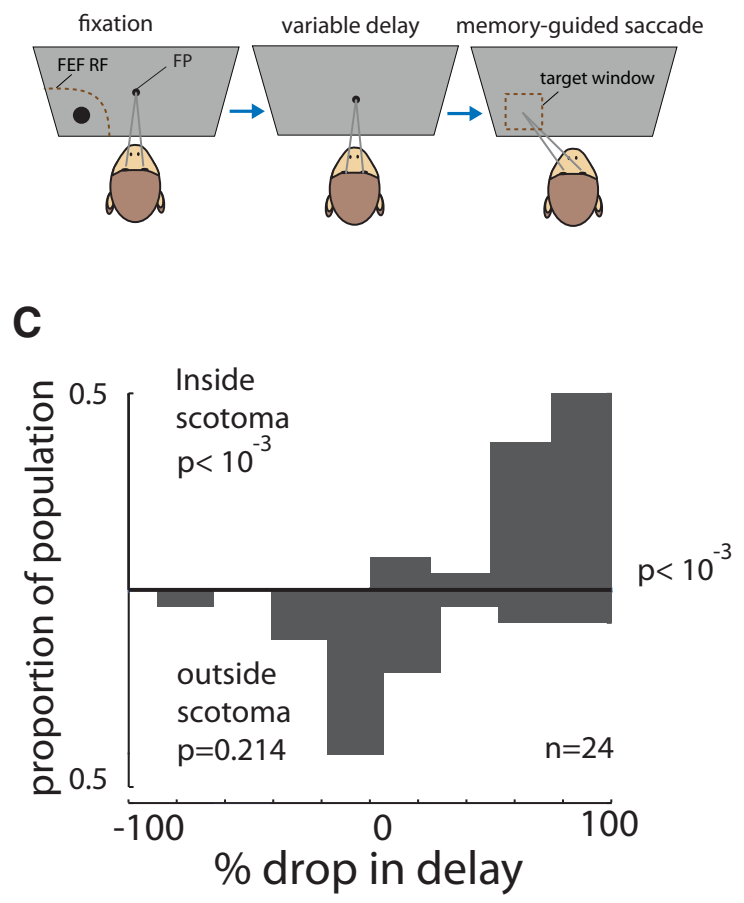

B

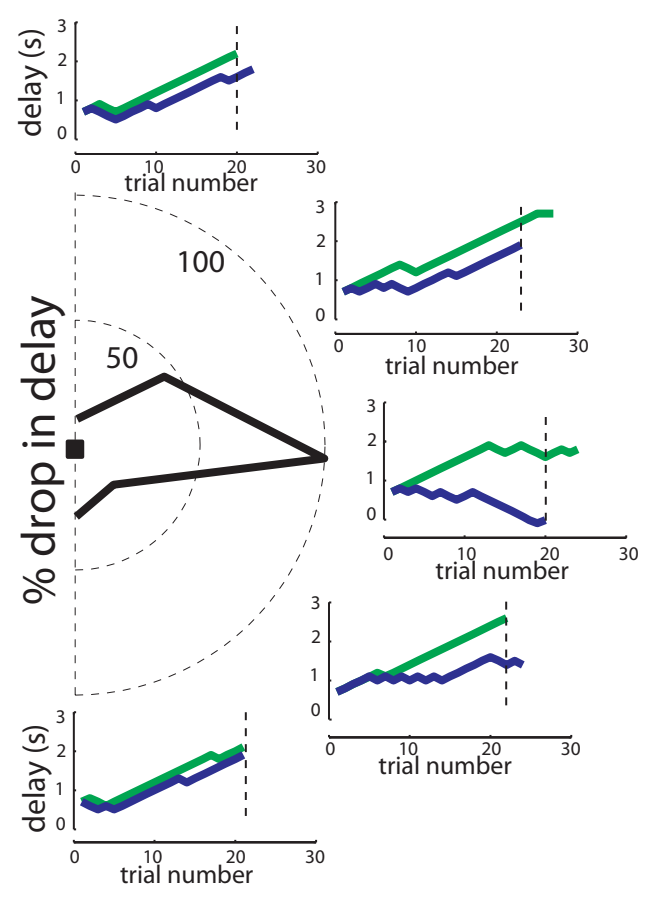

Figure 2. Effects of FEF inactivation on memory-guided saccades. $A$, In the memory-guided saccade task, the monkey is required to remember the location of a cue (black circle) across a variable delay and to make a saccade to the remembered cue location after the go signal (fixation spot offset). $\boldsymbol{B}$, Performance of a monkey in a sample experiment for five different locations before and after FEF inactivation. The $y$-axis in each plot is the delay duration, adjusted separately at each location via a staircase procedure. In this procedure, a correct trial results in an increase in the delay duration for the next trial at that location, whereas an incorrect response reduces the delay for the next trial. Green and blue lines illustrate the performance before and after FEF inactivation, respectively. Steep staircase functions indicate an ability to perform the task at increasingly long delays. In contrast, flat or declining functions indicate relatively poor ability, or complete inability (e.g., blue in middle plot), to remember cue location for even very short delays. The black polar plot illustrates the percentage drop in delay due to inactivation measured after an equal number of trials in each location (dashed line in each plot). C, Distribution of percentage drop in delay for 24 inactivation experiments for cue locations inside the scotoma (top) and location $90^{\circ}$ (theta) away from that location (outside scotoma, bottom).

crease in the delay. Thus, the monkey had to correctly complete trials to receive any reward. As shown in Figure $2 B$, the monkey was able to complete memory-guided saccades with delays as long as $2-3 \mathrm{~s}$ at most locations. For example, after 20 trials at the rightward location the monkey was completing trials with a delay of $2 \mathrm{~s}$. However, after inactivation, for the same number of trials, the staircase procedure resulted in a $0 \mathrm{~s}$ delay; a $100 \%$ drop in the final delay duration. As shown in the polar plot in Figure $2 B$, this drop is maximal for the horizontal location and becomes gradually smaller for flanking locations. This spatial specificity of the infusion-induced deficit in memory guided saccade performance was observed across 24 inactivation experiments in two monkeys (Fig. 2C). The most severely affected location showed a significant drop in delay duration (percentage drop inside scotoma $=77 \pm 26 \%, p<10^{-3}$ ), but performance was not significantly impaired at locations $90-180^{\circ}$ away (percentage drop outside scotoma $=11 \pm 42 \%, p=0.214$; inside vs outside scotoma comparison, $p<10^{-3}$ ). Thus, the inactivation of the FEF led to deficit in the memory guided saccade task that was concentrated in a localized portion of the contralateral visual field.

Analyses of V4 activity. All data analysis was performed in MATLAB (MathWorks). Only completed trials were included in the analysis. Summarized below are the several ways in which V4 activity was quantified.

Time windows. FEF and V4 presaccadic responses were calculated in a $125 \mathrm{~ms}$ window starting $100 \mathrm{~ms}$ before saccade onset; this window was chosen based on previous studies of presaccadic activity in extrastriate cortex (Fischer and Boch, 1981a,b; Moore et al., 1998). The fixation portion of the response was defined as the response in a $1000 \mathrm{~ms}$ window starting $40 \mathrm{~ms}$ after stimulus onset; however, for comparisons between activity during the presaccadic period and the fixation period, the fixation period is defined as a $125 \mathrm{~ms}$ window right before the go cue (fixation spot offset). The tonic activity was defined within a $500 \mathrm{~ms}$ window beginning $300 \mathrm{~ms}$ after stimulus onset. Baseline activity was defined as the activity during $0-300 \mathrm{~ms}$ before stimulus onset.

Response normalization. The responses of each V4 neuron to RF stimuli were converted to normalized values for analyses. Specifically, each neuron's response to each stimulus was divided by its grand average response to both preferred and nonpreferred stimuli before and after drug infusion. For FEF recordings, neuronal responses recorded before and after infusion were treated as independent samples (due to potential volume effects of the infusion), and normalized separately. Normalized values ranged between 0 and 1 according to following formula:

Normalized response $=$ (response

$$
-\min (\text { response })) /(\max (\text { response })-\min (\text { response })) .
$$

Where min and max responses are the peak and trough of the responses during the baseline and fixation periods. Normalized responses are indicated by NU (normalized units) in the text.

Discriminability. A receiver operating characteristic (ROC) analysis was performed on the distributions of firing rates of V4 neurons during the RF presentation of the most effective oriented bar stimulus and an orthogonal bar during a given block of trials. The areas under ROC curves were used as an index of stimulus selectivity and were calculated as in previous studies (Britten et al., 1992), using the presaccadic and fixation time windows defined above.

Selectivity index. As in previous studies (Monier et al., 2003), a selectivity index was computed as the difference in response to the preferred and the response to the nonpreferred stimulus divided by their sum, during the presaccadic and fixation windows defined above. For the analysis described in Figure 9, in which correlations between preferred 

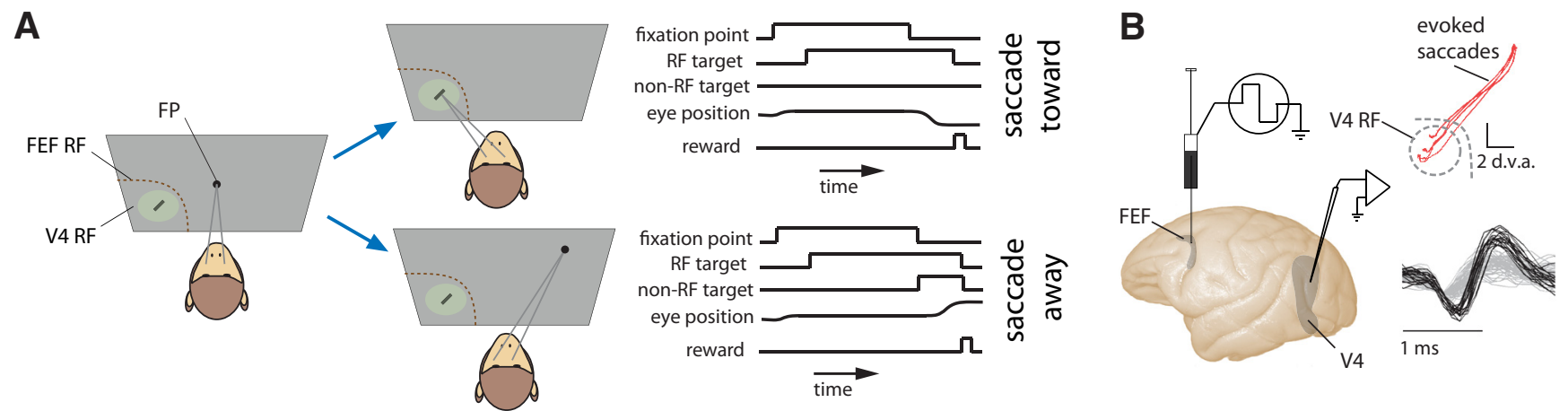

Figure 3. Simultaneous inactivation of the FEF and recordings from area V4 neurons during a visually guided saccade task. $\boldsymbol{A}$, Visually guided, delayed saccade task in which monkeys made delayed saccades to visual stimuli (targets) appearing within the RF of V4 neurons under study (saccade toward) or to a distant non-RF target (saccade away). Event plots to the right show the sequence of trial events. FP, fixation point. $\boldsymbol{B}$, A microinjectrode was used to elicitsaccades (red traces) with microstimulation and record from FEF neurons before and after local infusion of muscimol. Recordings were made from V4 neurons with RFs within the part of space affected by the FEF inactivation.

and nonpreferred response differences and changes in average presaccadic activity were computed for each neuron separately, the selectivity index was computed only as preferred-nonpreferred (to avoid introducing a false correlation by normalizing by a value, then calculating a correlation with that same value).

The surround suppression index (SSI) was computed as (presurround - postsurround)/(presurround + postsurround) using the firing rate measured during two $65 \mathrm{~ms}$ time windows, one shortly before (presurround) and one after (postsurround) surround onset. The presurround window began $100 \mathrm{~ms}$ before surround onset, and the postsurround window began $50 \mathrm{~ms}$ after the surround onset.

Subsampling analysis. For the analysis in Figure 9, the change in average response and stimulus selectivity was compared between the final 125 $\mathrm{ms}$ of the fixation period and a $125 \mathrm{~ms}$ presaccadic window aligned to saccade onset $(-100$ to $+25 \mathrm{~ms})$. We were interested in determining the nature of the relationship between the change in average response, defined as (preferred + nonpreferred) $/ 2$, and selectivity, defined as (preferred-nonpreferred), for each neuron. To understand this relationship, we used the bootstrapping technique (Efron and Tibshirani, 1993). In this approach, each subsample contains the same number of trials as the full dataset (which varied in size from day to day, with a minimum of 20 trials/condition), constructed by random sampling with replacement from the original dataset; therefore, one trial can be used more than once in each subsample whereas another trial is omitted. One-thousand subsamples were used to calculate the correlation value for each neuron. Notice that the reason the correlation cannot be performed across single trials is that quantification of selectivity requires at least two trials. In addition to the subsampling approach reported in Figure $9 A, B$, we examined the correlation between the average response and stimulus selectivity on individual pairs of trials (Fig. 9C). To do so, we paired each preferred stimulus trial with a nonpreferred stimulus trial. Pairings were based on temporal proximity of the two trials; i.e., each preferred trial was paired with the nonpreferred trial that was closest in time and not already assigned to a pair. We then calculated the average response and stimulus selectivity for each pair of trials, and the correlation between these two measures was determined across pairs. This trial-pairing method reproduced all the main results of the subsampling method.

Statistical tests. A criterion level of $p<0.05$ was used in all statistical analysis. $P<0.001$ are reported as $p<10^{-3}$. We used Wilcoxon signedrank tests to evaluate the statistical significance of differences between two paired populations or whether a single population's median differed from zero. Other tests used throughout the text are mentioned in each instance. Correlations were measured using the Pearson correlation method.

Behavioral tasks. During each recording experiment, monkeys performed a visually guided, delayed saccade task (Fig. $3 A$ ). After fixating a 0.5 dva fixation spot within a 1.0 dva diameter window, an oriented bar appeared within the RF of the V4 neuron being recorded. Following a $1 \mathrm{~s}$ delay, monkeys made saccades to visual stimuli (targets), presented ei- ther within the RF of V4 neuron ("saccade toward", $50 \%$ of trials) or to a distant non-RF target ("saccade away," 50\% of trials); the two conditions were randomly interleaved so that the monkey could not predict the saccade location until after the removal of the fixation spot. In addition, the effects of FEF inactivation on behavior was assessed with a memory guided saccade task, in which monkeys had to remember the location of a visual target, and then make saccades to its location after a variable delay (Fig. 2A).

In 15 of 19 inactivation experiments, we briefly presented $(13 \mathrm{~ms})$ a surround stimulus $(1 \times 1$ dva white circle $)$ at a random time $(200-800$ ms after RF stimulus onset). The surround stimulus was placed at a random location, always at least 6 dva away from the RF stimulus, but otherwise anywhere on the screen.

\section{Results}

\section{Effects of muscimol on FEF neural activity}

At 19 sites within the FEF of two monkeys, we infused $0.5-1.0 \mu \mathrm{l}$ of the GABAa agonist muscimol $(5 \mathrm{mg} / \mathrm{ml})$. At the beginning of each experiment, before drug infusion, we first confirmed the location of the microinjectrode within the FEF by evoking saccades with low-current electrical microstimulation (see Materials and Methods). The endpoints of the evoked saccadic vectors also defined the part of retinotopic space represented by neurons at the stimulation site (Fig. 3B). Next, we identified area V4 neurons with RFs that corresponded retinotopically to the site of FEF inactivation; we recorded these V4 neurons before and after FEF inactivation.

Although our primary focus was on the effects of FEF inactivation on area V4 neuronal responses, we occasionally recorded FEF neuronal responses after the local infusion of muscimol. Figure $4 A$ shows the multiunit activity of neurons at a sample FEF site before and after the infusion of $0.5 \mu \mathrm{l}$ of muscimol. Following the infusion, we observed a significant decline in baseline firing rate (mean \pm SEM baseline response: control $=40 \pm 1$ spikes $/ \mathrm{s}$; muscimol $=15 \pm 1$ spikes/s) and a reduction in visual and presaccadic responses (visual response: control $=63 \pm 1$ spikes $/ \mathrm{s}$; muscimol $=17 \pm 0.5$ spikes/s; presaccadic response in toward condition: control $=103 \pm 5$ spikes/s; muscimol $=22 \pm 2$ spikes/s; presaccadic response in away condition: control $=91 \pm$ 6 spikes/s; muscimol $=26 \pm 3$ spikes/s). We did not assume that the postinfusion responses were from the same neurons recorded before inactivation, given the likely disruption in recording stability with volume injections. Instead, we treated the preinactivation and postinactivation data as different (unpaired) neuronal populations, and normalized their activity separately (see Materials and Methods). Nonetheless, we could observe that with a 


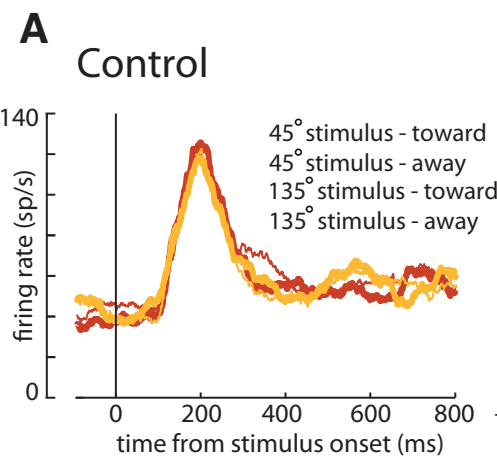

B

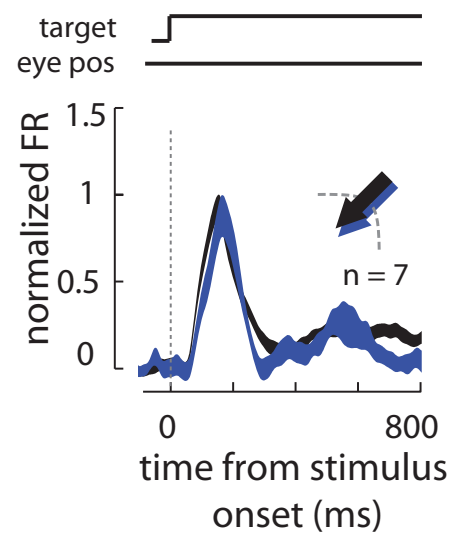

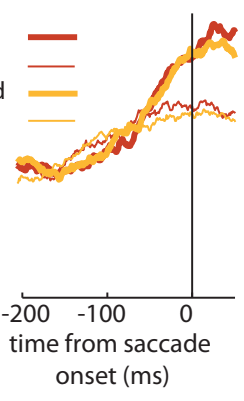

C

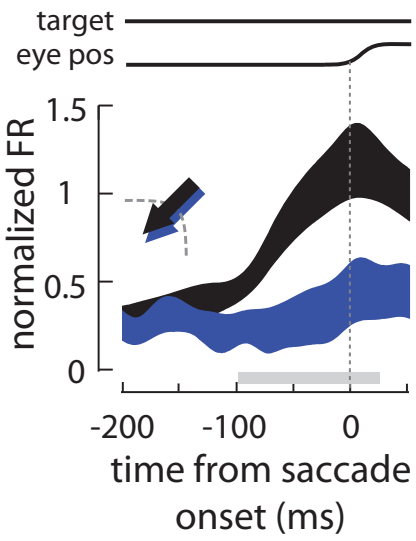

Muscimol infusion

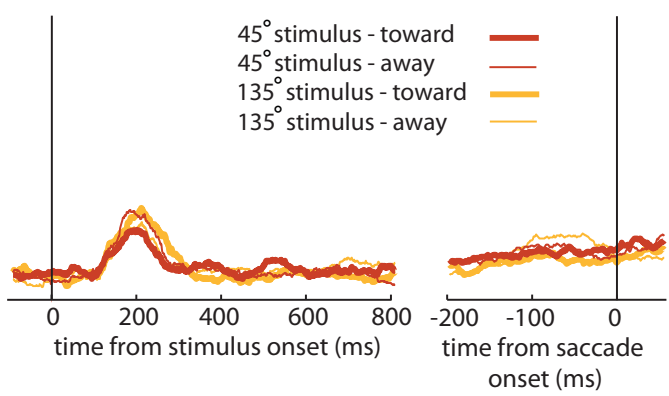

D

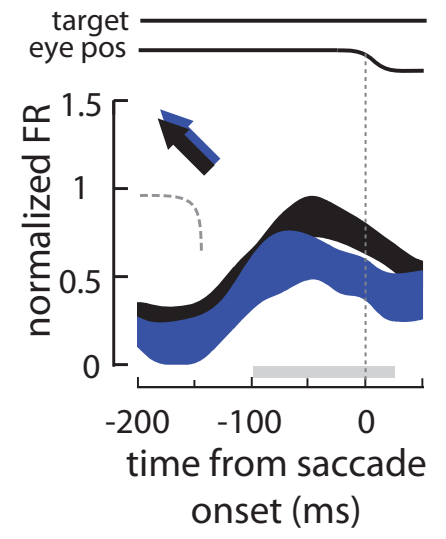

Figure 4. Inactivation of FEF activity. $\boldsymbol{A}$, Visual and saccade related activity recorded at an FEF site before (left) and after (right) local infusion of $0.5 \mu$ l of muscimol. Control and postinfusion mean responses are aligned first to stimulus onset (left) and then to saccadic onset (right) at the end of the behavioral trial. Note the absence of orientation selectivity but the clear presence of a presaccadic enhancement that is eliminated following the infusion of $0.5 \mu$ l of muscimol. $\boldsymbol{B}$, Mean normalized firing rate (FR) of FEF neurons to visual stimuli before infusion (black), and $>30$ min after the muscimol infusion (blue). Each response is aligned to visual stimulus onset (above traces). Arrows indicate eye movements into or away from the FEF RF for the corresponding plot. $\boldsymbol{C}$, Presaccadic responses for samples of FEF neurons for saccades made to targets in their RFs. Responses are aligned to saccadic onset. $D$, Presaccadic responses for saccades made to targets outside of the FEF RFs. Gray bars on bottom denote the presaccadic time window used in the analysis.

small number of residually responsive FEF neurons $(n=7)$, the inactivation reduced the strength of tonic visual activity relative to the initial visual response $(n=7 ; \Delta$ response $=-0.08 \pm 0.04$ $\mathrm{NU}, p=0.006$; Wilcoxon rank sum test; Fig. $4 B$ ). More importantly, muscimol dramatically reduced presaccadic responses (relative to the visual response) for saccades made to RF targets in this sample of neurons ( $\Delta$ presaccadic response $=-0.57 \pm 0.15$ NU, $p=0.001$; Fig. $4 C$ ). Presaccadic responses appeared unchanged for saccades made to non-RF locations ( $\Delta$ presaccadic response $=-0.21 \pm 0.13 \mathrm{NU}, p=0.216$; Fig. $4 D$ ). Local infusion of saline neither changed the tonic visual activity $(\Delta$ response $=$ $0.01 \pm 0.04 \mathrm{NU}, p=0.639 ; n=4)$ nor the presaccadic responses for saccades made toward $(\Delta$ presaccadic response $=0.03 \pm 0.13$ NU, $p=0.875$ ) or away from RF targets ( $\Delta$ presaccadic response $=0.01 \pm 0.09 \mathrm{NU}, p=0.882)$. Thus, we observed direct evidence that muscimol strongly suppressed presaccadic activity within the FEF.

\section{Effects of FEF inactivation on V4 neuronal responses}

Contrary to our expectations, we found that the magnitude of presaccadic activity in V4 increased following FEF inactivation. Although the magnitude of presaccadic activity in V4 increased, orientation selectivity of V4 responses during this period decreased. These effects are illustrated in the responses of an example V4 neuron with an RF overlapping that of the FEF site (Fig. 5). The responses of this neuron are shown before and after infusion
(Fig. 5A, $B$, respectively), aligned to the onset of the visual stimulus (left), and to the time of the saccade (right), for preferred (black, orange), and nonpreferred stimuli (gray, peach; Fig. $5 A, B$, respectively). Only trials in the saccade toward condition are included. This V4 neuron displayed significant orientation selectivity: a $45^{\circ}$ oriented bar evoked $25.07 \pm 1.13$ spikes $/ \mathrm{s}$ in this neuron whereas a $135^{\circ}$ stimulus evoked $13.87 \pm 0.63$ spikes $/ \mathrm{s}$ (preferred vs nonpreferred orienation; Wilcoxon rank sum test; $\left.p<10^{-3}\right)$. The neuron also exhibited enhanced activity during the presaccadic period relative to the tonic visual response (for saccades into the RF), for both preferred and nonpreferred stimuli (presaccadic enhancement preferred $=6.72 \pm 2.56$ spikes $/ \mathrm{s}, p=$ 0.028; presaccadic enhancement nonpreferred $=5.66 \pm 1.67$ spikes/s, $p=0.006)$. FEF inactivation resulted in an increase in this presaccadic enhancement for both preferred and nonpreferred stimuli ( $\Delta$ presaccadic enhancement preferred $=2.62$ spikes $/ \mathrm{s}, p=0.01 ; \Delta$ presaccadic enhancement ${ }_{\text {nonpreferred }}=3.12$ spikes/s, $p=0.006$; Fig. $5 A, B)$. However, following FEF inactivation the orientation selectivity of the cell decreased during the same presaccadic period: the difference between preferred and nonpreffered responses was reduced after FEF inactivation $[\Delta$ (preferred - nonpreffered $)=-3.03 \pm 1.01$; Wilcoxon rank sum test; $p=0.02]$. The difference between responses to preferred and nonpreferred stimuli was also reduced during the visual period $[\Delta$ (preferred - nonpreffered $)=-3.61 \pm 0.82$, Wilcoxon rank sum test; $p=0.03$ ]; however, average visual ac- 
A

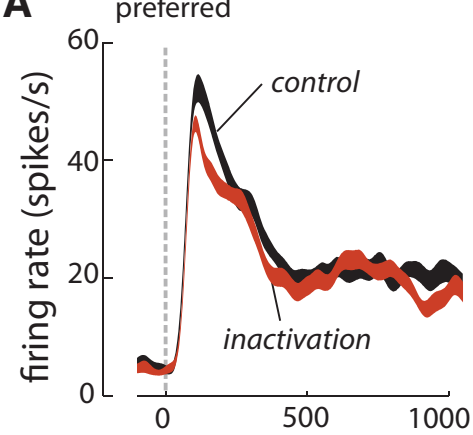

B

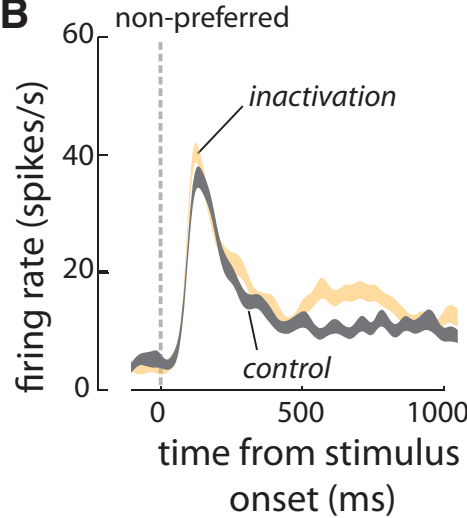

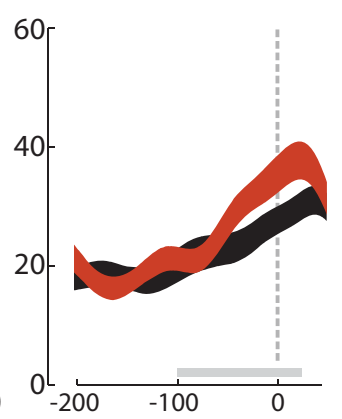

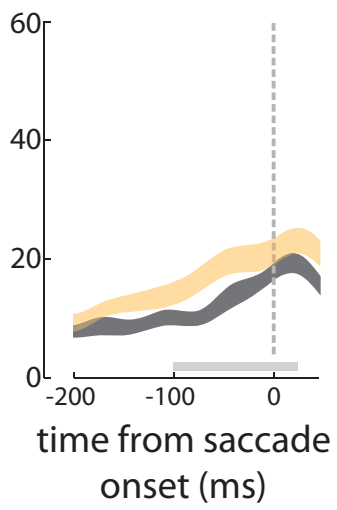

Figure 5. Effects of FEF inactivation on the responses of an exemplar V4 neuron. $\boldsymbol{A}$, Visual responses of one V4 neuron to its preferred stimulus before (black) and after FEF inactivation (orange). Responses are shown aligned to the onset of the visual stimulus (left axis), and to the onset of the saccade (right axis), for saccades toward the V4 RF only. $\boldsymbol{B}$, Responses of the same V4 neuron to its nonpreferred stimulus before (gray) and after FEF inactivation (peach).

tivity during the visual period (mean response to preferred and nonpreferred stimuli) was not altered by FEF inactivation $(\Delta$ average activity $=-1.61 \pm 0.82$; Wilcoxon rank sum test; $p=$ 0.32 ). Thus, for the example V4 neuron presented, FEF inactivation increased presaccadic enhancement but reduced orientation selectivity during the visual and presaccadic periods.

Similar effects were observed when we tested the effects of FEF inactivation on the responses of $33 \mathrm{~V} 4$ neurons with RFs overlapping those of FEF neurons at the inactivation site (Fig. 6A). The centers of the V4 and FEF RFs were separated by an average of $1.11 \pm 0.11 \mathrm{dva}$, which is only a fraction of RF diameter in these two areas (Wurtz and Mohler, 1976; Gattass et al., 1988). Baseline activity of V4 neurons was not altered by inactivation of FEF sites with corresponding RFs. Mean baseline activity was $0.28 \pm 0.04$ NU before FEF inactivation, and $0.28 \pm 0.03 \mathrm{NU}$ after inactivation (baseline activity, control vs inactivation; $p=0.52$ ). We defined the presaccadic enhancement as the change in neuronal activity before saccade onset, comparing firing rates during the presaccadic period with those during the fixation period, and quantified it using ROC analysis. Before FEF inactivation, V4 presaccadic enhancement was significantly $>0.5$ for saccades to stimuli in the RF (toward condition), reflecting the presaccadic increase in firing rate $\left(n=33 ; \mathrm{AROC}_{\text {toward,control }}=0.56 \pm 0.01\right.$, $p<10^{-3}$; Fig. $6 B$ ). In contrast, saccades to stimuli outside of the $\mathrm{RF}$ (away condition) resulted in a reduction of V4 activity (ARO$\mathrm{C}_{\text {away,control }}=0.48 \pm 0.01, p<10^{-3}$ ). Thus, as previously shown, V4 neurons signal the direction of impending saccades (Fischer and Boch, 1981b; Moore et al., 1998). We also quantified the ability of V4 neurons to signal impending saccade direction using

A
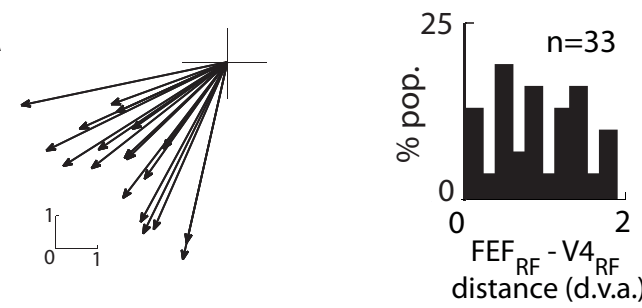

B

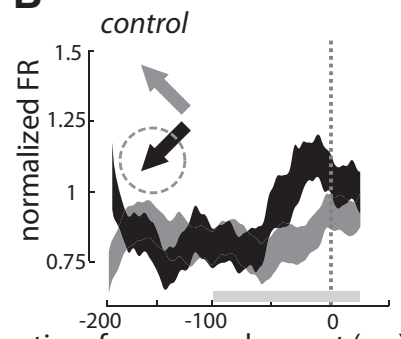

C

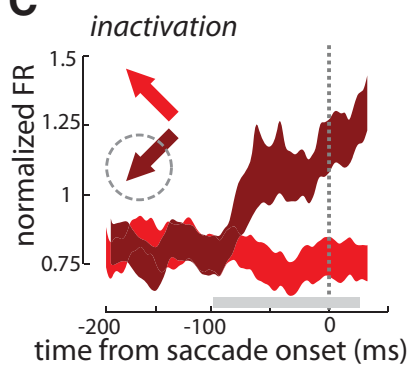

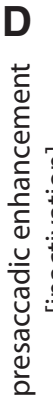

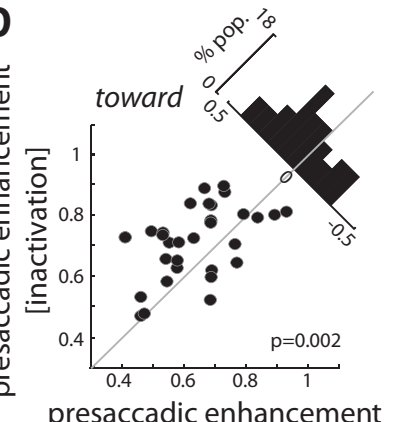

[control]

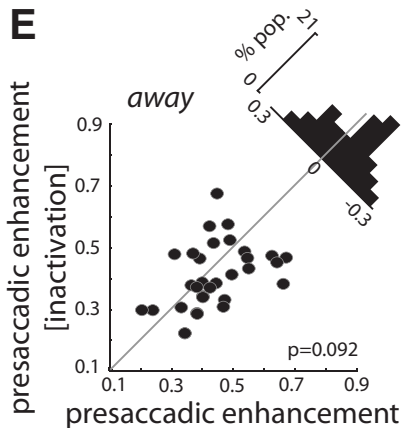

[control]

Figure 6. Effects of FEF inactivation on presaccadic enhancement in V4. A, Recordings were made from $33 \mathrm{~V} 4$ neurons with RFs within the part of space affected by the FEF inactivation. Left, evoked saccade vectors of FEF sites before inactivation. Right, distribution of distances between the FEF RF and each V4 RF. B, V4 presaccadic responses for saccades made to RF stimuli (black) or to targets outside of the RF (gray) before inactivation. Arrows indicate eye movements into or away from the V4 RF for the corresponding plot. C, V4 presaccadic responses for saccades made to RF stimuli (dark red) or to targets outside of the RF (bright red) after inactivation. D, Comparison of presaccadic enhancement (AROC) for saccades made into the RF (toward) before and after FEF inactivation. Histogram on the diagonal shows the difference in enhancement. $\boldsymbol{E}$, Comparison of presaccadic enhancement for saccades made outside the RF (away) before and after FEF inactivation.

the ROC analysis (toward vs away; $\mathrm{AROC}_{\text {control }}=0.58 \pm 0.01$, $p<10^{-3}$ ).

Our hypothesis was that inactivation of the FEF would eliminate or reduce presaccadic enhancement in area V4. However, contrary to our prediction, we found that FEF inactivation instead increased the magnitude of presaccadic enhancement (Fig. 6C). We observed greater presaccadic enhancement in the population of recorded V4 neurons following the FEF inactivation compared with the control data $\left(\Delta \mathrm{AROC}_{\text {toward }}=0.05 \pm 0.01, p<10^{-3}\right.$; Fig. $\left.6 D\right)$. In addition, we observed a trend toward greater suppression of presaccadic responses in the away condition $\left(\Delta \mathrm{AROC}_{\mathrm{away}}=-0.016 \pm 0.01, p=\right.$ 0.09 ; Fig. $6 E$ ). Both effects contributed to an overall increase in the degree to which V4 neurons signaled the direction of impending saccades $\left(\Delta \mathrm{AROC}_{\text {toward vs away }}=0.07 \pm 0.01, p<10^{-3}\right)$.

Despite the increase in presaccadic enhancement following FEF inactivation, we observed no change in the average tonic visual response. The average visual response of the population of 
A

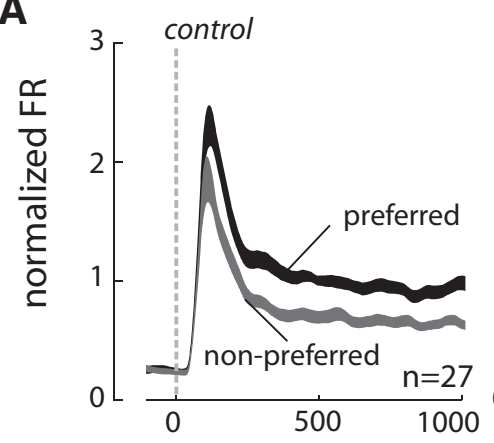

B

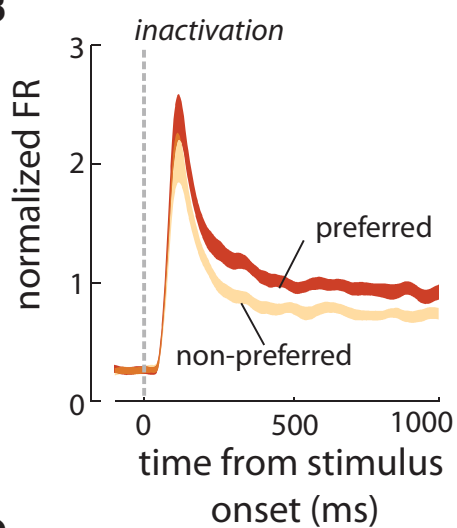

C

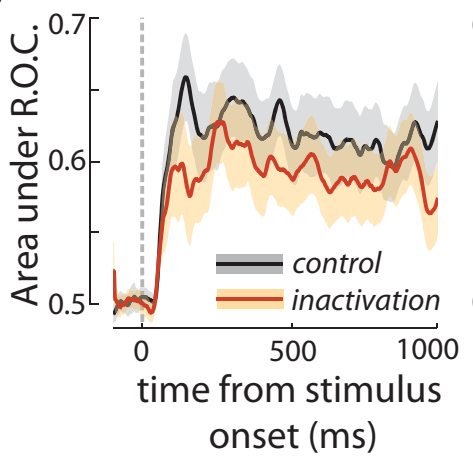

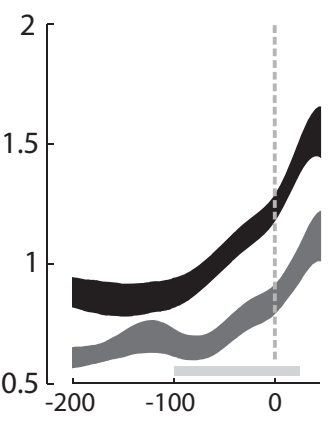
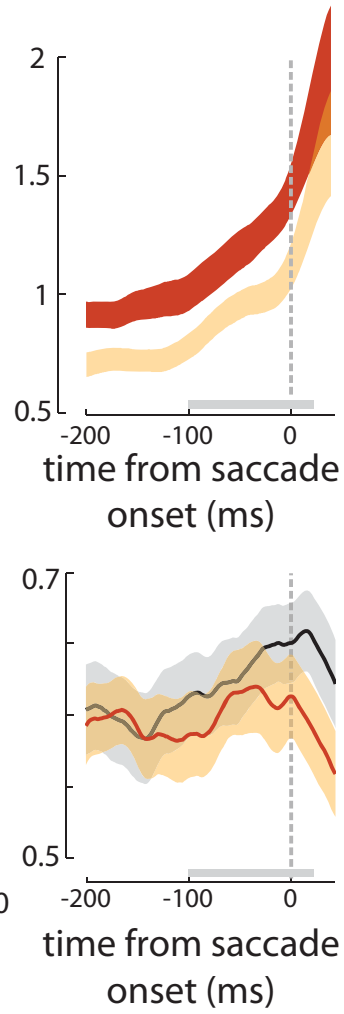

Figure 7. Effects of FEF inactivation on V4 responses to preferred and nonpreferred stimuli. $\boldsymbol{A}$, Visual responses of 27 stimulus-selective V4 neurons to preferred (black) and nonpreferred bar orientations (gray) before FEF inactivation. Responses are shown aligned to the onset of the visual stimulus (left axis), and to the onset of the saccade (right axis), for saccades toward the V4 RF only. $\boldsymbol{B}$, Responses of the same $27 \mathrm{~V} 4$ neurons after FEF inactivation, for preferred (orange) and nonpreferred stimuli (peach). C, Selectivity of V4 visual responses, measured as the area under an ROC curve, before FEF inactivation (black) and after FEF inactivation (orange); responses are shown aligned to stimulus onset (left axis) and saccade initiation (right axis). ROC area calculated in a sliding $50 \mathrm{~ms}$ window.

V4 neurons was $0.99 \pm 0.19 \mathrm{NU}$ before and $1.01 \pm 0.29 \mathrm{NU}$ after FEF inactivation $(n=33 ; p=0.809)$.

Of the recorded neurons, 27 significantly discriminated RF stimulus orientation. V4 visual responses showed nonsignificant trends toward increased responses to nonpreferred stimuli and decreased responses to preferred stimuli (Fig. $7 A, B$; $n=27 ; \Delta$ preferred $=-0.03 \pm 0.07 \mathrm{NU}, p=0.648$; $\Delta$ nonpreferred $=0.09 \pm 0.06 \mathrm{NU}, p=0.102$ ). These trends combined to produce a significant reduction in the stimulus (orientation) selectivity index of V4 visual responses following FEF inactivation ( $\Delta$ selectivity index $=-0.04 \pm 0.01, p=$ $0.002)$. As in previous studies, the selectivity index was defined as the difference between responses to preferred and nonpreferred stimuli divided by their sum (Monier et al., 2003). We

A
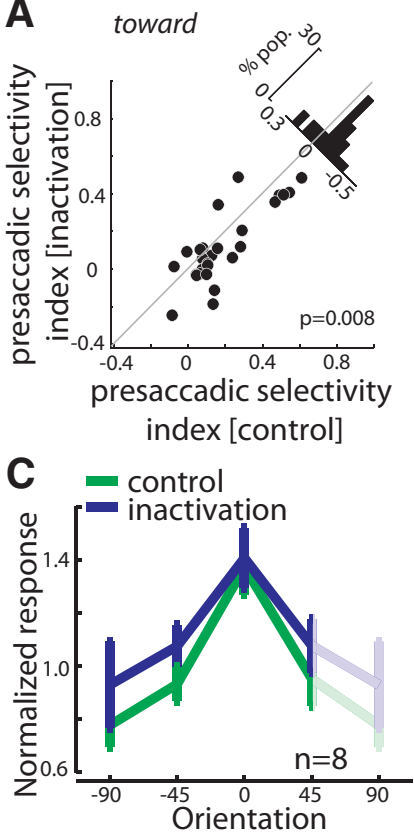

(relative to preferred stimulus)

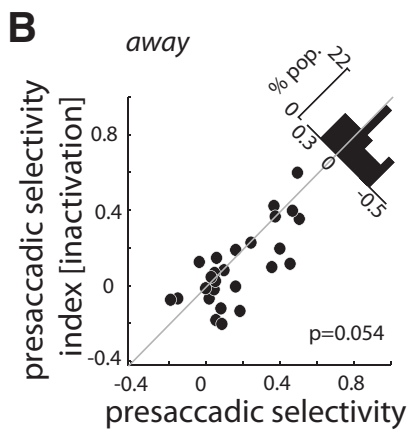

index [control]

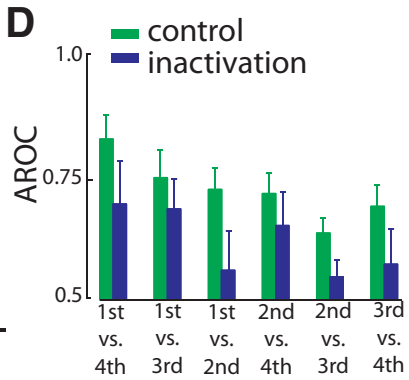

Stimulus pair
Figure 8. Effects of FEF inactivation on V4 selectivity indices and tuning. $\boldsymbol{A}$, Comparison of presaccadic stimulus selectivity indexes for saccades made into the RF (toward) before and after FEF inactivation. Histogram on the diagonal shows the difference in selectivity index. $\boldsymbol{B}, \mathrm{Com}-$ parison of presaccadic stimulus selectivity indexes for saccades made outside the RF (away) before and after FEF inactivation. C, Effect of inactivation on the orientation tuning of V4 neurons. The normalized responses of $8 \mathrm{~V} 4$ neurons to four different orientations (relative to the preferred) are shown (dark lines) before (green) and after inactivation (blue). Data from $-90^{\circ}$ is duplicated at $+90^{\circ}$ for visualization purposes (light lines). $D$, FEF inactivation reduces orientation discriminability (AROC) during the presaccadic period. Bar plot shows the mean discriminability for all six pairwise comparisons of responses to the 4 orientations before (green) and after FEF inactivation (blue). The four stimulus orientations were rank ordered from first to fourth optimal based on the evoked response.

also measured the ability of V4 responses to discriminate RF stimuli (stimulus discriminability) using an ROC analysis, which takes into account the variability of visual responses as well as the difference in their magnitude. This analysis revealed that the ability of V4 neurons to discriminate between preferred and nonpreferred stimulus orientations is reduced by $24 \%$ after FEF inactivation $\left(\mathrm{AROC}_{\text {control }}=0.68 \pm 0.02\right.$; $\mathrm{AROC}_{\text {inactivation }}=0.64 \pm 0.02 ; \Delta \mathrm{AROC}=-0.04 \pm 0.01, p<$ $\left.10^{-3}\right)$; the full time course of this decrease in discriminability can be seen in Figure $7 C$. The inactivation-induced reduction in stimulus discriminability observed during the tonic visual response was also present during the presaccadic period, the period during which presaccadic enhancement actually increased. Figure $7 A, B$ shows the presaccadic responses of the population of V4 neurons before and after FEF inactivation for the toward condition. Following inactivation, neuronal activity during the presaccadic period increased both for preferred and nonpreferred RF stimuli ( $\Delta$ preferred $=0.15 \pm 0.07$ $\mathrm{NU}, p=0.043 ; \Delta$ nonpreferred $=0.20 \pm 0.06 \mathrm{NU}, p=0.002)$. However, the difference between the responses to the preferred and nonpreferred stimuli was reduced during this pe$\operatorname{riod}(\Delta$ selectivity index $=-0.06 \pm 0.02, p=0.008 ;$ Fig. $8 A$ ). In the away condition, we also observed a nonsignificant trend toward a reduction in the selectivity index and stimulus discriminability $(n=27 ; \Delta$ selectivity index $=-0.06 \pm 0.027$, $p=0.054 ; \Delta \mathrm{AROC}=-0.04 \pm 0.02, p=0.079$; Fig. $8 B)$. 
A

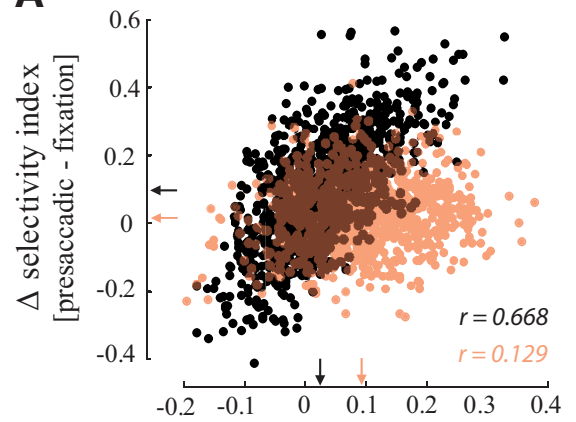

$\Delta$ average response [presaccadic - fixation]
B

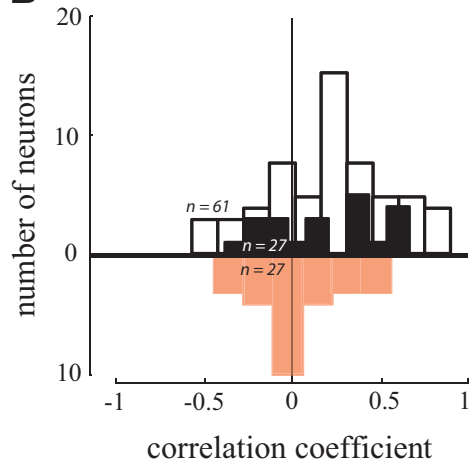

C

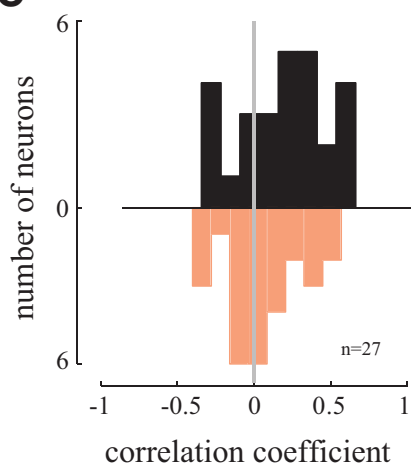

Figure 9. The relationship between presaccadic enhancement and the stimulus selectivity index of V4 neurons. $\boldsymbol{A}$, Correlation between presaccadic changes in average response and stimulus selectivity for an example V4 neuron before (black) and after (salmon) inactivation. Each point represents changes in average response (preferred and nonpreferred) and stimulus selectivity index (preferred vs nonpreferred) computed for a randomly selected subset of trials. Arrows indicate the marginal means. Pearson correlation coefficients ( $r$ ) computed for data before and after inactivation are shown. $\boldsymbol{B}$, Distributions of correlation coefficients for all $27 \mathrm{~V} 4$ neurons recorded before (black) and after FEF inactivation (salmon), calculated using the subsampling method illustrated in $\boldsymbol{A}$. The distribution of correlation coefficients for a population of 61 V4 neurons from a previous study (Moore and Chang, 2009; white) is also shown. $\boldsymbol{C}$, Distributions of correlation coefficients for the same 27 V4 neurons recorded before (black) and after FEF inactivation (salmon), calculated using the trial-pairing method.

The reduction in the stimulus selectivity index during the presaccadic period was also apparent in a subset of eight neurons studied using four different stimulus orientations (Fig. 8C). Stimulus discriminability, as measured by the ROC analysis in the toward condition, was reduced following the FEF inactivation $(\triangle \mathrm{AROC}=$ $-0.04 \pm 0.01, p=0.026)$. In addition, we observed that for the eight neurons tested with four different stimulus orientations, the inactivation-induced reduction in discriminability was independent of which pair of stimulus responses were compared (Fig. $8 D$; twoway ANOVA, inactivation effect: $F=8.93, p=0.003$; orientation pair: $F=3.05, p=0.012$; interaction: $F=0.23, p=0.949$ ). Thus, although FEF inactivation increased the ability of V4 neurons to signal the direction of impending saccades, it reduced their ability to discriminate the orientation of the target. We also separately examined the effect of FEF inactivation on the variability of V4 visual responses. Variability was quantified as the variance in spike counts across trials divided by their mean (Fano factor; Churchland et al., 2010). FEF inactivation did not significantly change the variability of V4 responses to preferred or nonpreferred stimuli $(n=27 ; \Delta$ Fano factor $_{\text {preferred }}=0.001 \pm 0.09, p=0.630 ; \Delta$ Fano factor $r_{\text {nonpreferred }}=$ $0.02 \pm 0.07, p=0.532$ ). The lack of an effect of FEF inactivation on response variability thus indicates that the observed reduction in stimulus discriminability resulted primarily from a decrease in the difference between the magnitude of responses to preferred and nonpreferred orientations. Last, to determine whether the observed changes in V4 responses were simply due to infusing a volume into the FEF, we also tested the effects of infusing the vehicle (saline) instead of muscimol. Saline infusions failed to alter the magnitude of presaccadic enhancement $\left(n=27 ; \Delta \mathrm{AROC}_{\text {toward vs away }}=0.01 \pm\right.$ $0.07, p=0.626)$ and failed to alter stimulus discriminability either during fixation $(\triangle \mathrm{AROC}=-0.01 \pm 0.013, p=0.380)$ or during the presaccadic period $(\Delta \mathrm{AROC}=0.01 \pm 0.02, p=0.331)$.

Without inactivation, both the magnitude and the stimulus discriminability of V4 visual responses increase before saccades made to RF targets (Moore and Chang, 2009). We wished to determine whether there is a relationship between these two effects. As shown for an exemplar neuron (Fig. 9A), saccades into the RF enhanced the average response to RF stimuli (preferred and nonpreferred), compared with the response during fixation. The stimulus selectivity index also increased during the presaccadic period. Across trials, the change in the presaccadic enhancement was positively correlated with the change in stimulus selectivity index $(r=0.668$, $\left.p<10^{-3}\right)$. For this neuron, FEF inactivation significantly reduced this correlation $(r=0.129$; ANCOVA, factors preinactivation vs postinactivation, presaccadic enhancement, presaccadic selectivity; main effect of FEF inactivation $p<10^{-3}$ ). We examined the relationship between presaccadic enhancement and the selectivity index in an additional population of 61 stimulus-selective V4 neurons from a previous study using the same visually guided saccade task (Moore and Chang, 2009). For this population, the mean correlation between presaccadic enhancement and the selectivity index was significantly greater than zero (Fig. 9B; $n=61 ; r=0.20 \pm 0.04, p<$ $\left.10^{-3}\right)$. This correlation was also present in the population of V4 neurons recorded before FEF inactivation in the current study $(n=27 ; r=0.21 \pm 0.05, p=0.001)$. Furthermore, the magnitude of this correlation was statistically equivalent between the two studies ( $p=0.763$; Wilcoxon rank sum test). Following FEF inactivation, the correlation between presaccadic enhancement and the selectivity index was eliminated $\left(r_{\text {inactivation }}=0.04 \pm 0.05, p=0.471\right)$ and this elimination rendered it significantly different from that of the control $(n=$ $\left.27 ; r_{\text {inactivation }- \text { control }}=-0.17 \pm 0.06, p=0.009\right)$. Baseline activity and selectivity values were subtracted from the presaccadic values in the preceding results to isolate changes in V4 activity around the time of a saccade. However, the same results also held when considering presaccadic activity and selectivity without subtracting out the baseline fixation values: presaccadic activity and selectivity were correlated before FEF inactivation $(r=0.21 \pm 0.05, p=0.001)$, but not correlated following inactivation $\left(r_{\text {inactivation }}=0.08 \pm 0.05, p=0.31\right)$, changing significantly between conditions ( $r_{\text {inactivation-control }}=-0.13 \pm 0.07, p=$ 0.019). FEF inactivation also eliminated the relationship between presaccadic enhancement and the selectivity index for saccades in the away condition $\left(r_{\text {control }}=0.16 \pm 0.05, p=0.009 ; r_{\text {inactivation }}=\right.$ $0.04 \pm 0.05, p=0.216 ; r_{\text {inactivation }}-$ control $=0.11 \pm 0.05, p=0.022$ ). We verified the findings of our subsampling approach using another analysis method, in which we paired each preferred stimulus trial with the closest nonpreferred stimulus trial (see Materials and Methods for details). The effects of FEF inactivation on the relationship between average activity and selectivity calculated using the trial- 

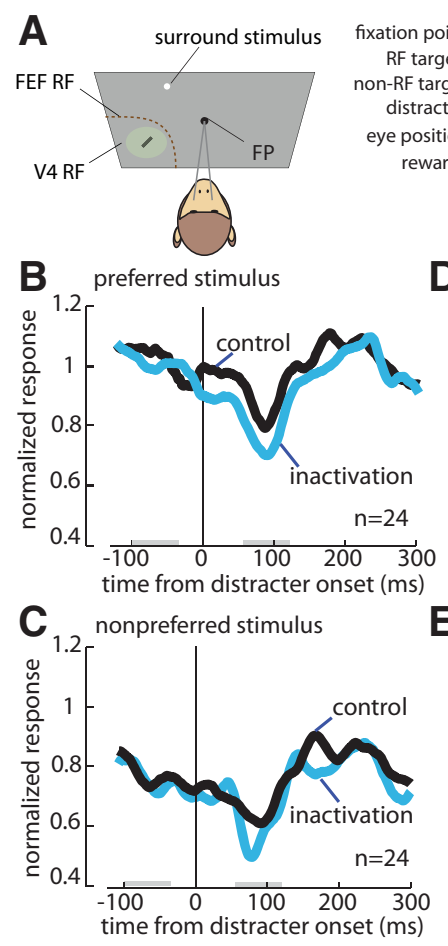

Figure 10. Effect of FEF inactivation on surround suppression of V4 responses. $\boldsymbol{A}$, Surround suppression paradigm: the same as the visually guided saccade paradigm previously described, except that a small surround stimulus appeared for $13 \mathrm{~ms}$ at a random location $>6 \mathrm{dva}$ from the receptive field stimulus at a random time $200-800 \mathrm{~ms}$ after the onset of the receptive field stimulus; timing was the same for toward and away conditions, illustrated here only for the toward condition. Gray shading indicates the time during which the surround stimulus could appear. $\boldsymbol{B}$, Surround stimulus-aligned response of a population of $24 \mathrm{~V} 4$ neurons to preferred stimuli before (black) and after FEF inactivation (blue). C, Surround stimulus-aligned response of the same population of V4 neurons to nonpreferred stimuli before (black) and after FEF inactivation (blue). D, Distributions of the SSIs for preferred stimuli (green) and nonpreferred stimuli (orange) before inactivation. $\boldsymbol{E}$, Distributions of the change in the SSIs for preferred and nonpreferred stimuli following FEF inactivation.

pairing method are shown in Figure 9C. Consistent with the results of the subsampling procedure, there was a significant correlation between activity and selectivity in the population of V4 neurons before FEF inactivation $\left(r_{\text {control }}=0.19 \pm 0.05, p=0.001\right)$. Also consistent with the results of the subsampling procedure, the correlation between presaccadic changes in average response and stimulus selectivity was eliminated by FEF inactivation $\left(r_{\text {inactivation }}=\right.$ $0.05 \pm 0.06, p=0.400$ ) and this elimination rendered it significantly different from that of the control $\left(r_{\text {inactivation-control }}=-0.14 \pm 0.06\right.$, $p=0.019)$. Therefore, FEF inactivation not only increased the magnitude of presaccadic enhancement and reduced stimulus selectivity index, but it also disrupted the normal relationship between these two measures.

In addition to testing the effect of FEF inactivation on V4 presaccadic activity, we measured its impact on surround suppression during fixation. Surround stimuli generally have suppressive influences on neuronal responses in area V4 (Desimone and Schein, 1987) and these effects interact with the locus of attention (Sundberg et al., 2009). In 15 of 19 inactivation experiments, we briefly presented $(13 \mathrm{~ms})$ a surround stimulus $(1 \times 1$ dva white circle) at a random time (200-800 ms after RF stimulus onset), at a location distant ( $>6 \mathrm{dva}$ ) from the RF (Fig. 10A). As shown in Figure 10, for the population of 24 V4 neurons, the surround stimulus presentation suppressed V4 neuronal responses (Fig. $10 B, C$, black traces; for preferred and nonpreferred stimuli respectively). Figure $10 \mathrm{D}$ shows the distribution of SSIs used to compare V4 activity before and after surround onset, positive SSIs indicating greater surround suppression. As shown, the SSI was significantly greater than zero both for preferred and nonpreferred stimuli $(n=24$; SSI preferred $=0.094 \pm 0.024, p<$ $10^{-3}$; SSI nonpreferred $\left.=0.122 \pm 0.030, p<10^{-3}\right)$, indicating a drop in activity following surround onset. Importantly, as shown in the blue trace in Figure 10B, after FEF inactivation, V4 responses showed even greater surround suppression. The SSI was $0.165 \pm 0.029$ for the preferred stimulus after FEF inactivation, reflecting a significantly larger surround-induced suppression of the $\mathrm{V} 4$ response $\left(\Delta S S_{\text {preferred }}=0.0713 \pm 0.024, p=0.009\right.$; Fig. $10 E)$. This change in the surround-induced response suppression was only observed when the preferred stimulus was presented within the V4 RF; there was no change in surround suppression of responses to nonpreferred stimuli after FEF inactivation $\left(\Delta \mathrm{SSI}_{\text {nonpreferred }}=-0.005 \pm 0.037, p=0.440\right.$; Fig. $10 \mathrm{C}$, blue trace, $E$, bottom histogram). Thus, following FEF inactivation, V4 neurons became more susceptible to suppressive influences from the surround, yet only when those neurons were adequately driven by RF stimuli.

\section{Discussion}

We examined the contribution of the FEF to presaccadic modulation in area V4 and found that, contrary to our expectations, FEF inactivation increased the magnitude of presaccadic enhancement. This increased enhancement was accompanied by a decrease in stimulus discriminability both during fixation and in the presaccadic period. Consequently, with reduced input from the FEF, V4 neurons signaled more about the direction of saccades, but less about the features of the saccadic target. FEF inactivation also disrupted the normal correlation between presaccadic changes in the response magnitude and the stimulus selectivity index. These results reveal the distinct contribution of the FEF to the visual cortical representation of saccadic targets.

\section{Sources of presaccadic enhancement in V4}

Presaccadic enhancement in V4 was altered following FEF inactivation, clearly indicating that the FEF plays some role in that enhancement. However, the persistence of presaccadic enhancement following a reduction in FEF input reveals that the FEF is not the sole source of that enhancement. In fact, not only did the enhancement persist during FEF inactivation, it increased in magnitude. What might account for this increase?

One straightforward explanation might be that FEF exerts a primarily inhibitory effect on V4 activity. However, key evidence argues against that explanation. First, a recent examination of FEF inputs to V4 found that a vast majority of the FEF input to V4 consists of excitatory synapses onto pyramidal neurons (Anderson et al., 2011). In addition, microstimulation of the FEF rapidly increases the magnitude and selectivity of V4 visual responses (Moore and Armstrong, 2003; Armstrong and Moore, 2007) suggesting that the direct projections from the FEF exert an excitatory, gain modulation on visually driven inputs to V4. Consistent with that evidence is the observed coherence and short phase lag between the spiking activity and local field potentials between the FEF and V4 (Gregoriou et al., 2009, 2012). Nonetheless, it might still be possible that the small subset of FEF inhibitory input to V4 neurons exerts a net inhibitory effect on presaccadic responses.

One possibility is that the elevated enhancement is due to a compensatory increase in presaccadic activity within other 
saccade-related structures; for example, the area LIP or the SC. Regarding the latter, it is known that both the FEF and the SC are necessary for normal saccadic eye movements, and that saccades are eliminated following combined lesions of both structures (Schiller et al., 1979, 1980). Furthermore, the SC projects inputs to extrastriate cortex via the pulvinar (Kaas and Huerta, 1988). However, recent data indicates that the tecto-pulvinar relay to V4 is weak or absent compared with dorsal extrastriate areas (e.g., MT; Lyon et al., 2010). These data suggest that a contribution of the SC to V4 presaccadic enhancement would need to be mediated by dorsal areas, including area LIP where neurons exhibit robust presaccadic modulation (Mazzoni et al., 1996). Recent work also provides some evidence of a compensatory relationship between FEF and SC. Berman et al. (2009) found that some FEF neurons exhibit increased presaccadic activity following inactivation of the SC. It could be that inactivation of the FEF similarly results in compensatory effects within the SC, and this compensation influences presaccadic enhancement in V4 via a tectopulvinar-dorsal stream route, perhaps involving area LIP. Alternatively, there could be a compensatory increase in presaccadic activity within LIP itself, and this compensation could be conveyed directly to V4 (Andersen et al., 1990).

Nevertheless, our results suggest that although there is a residual source of presaccadic enhancement, the FEF contributes uniquely to presaccadic modulation. The relationship between presaccadic enhancement and the presaccadic change in the stimulus selectivity index observed before inactivation suggests that information about the direction of upcoming saccades and about visual target features covary under normal circumstances in V4. We found that this relationship was eliminated by FEF inactivation. This finding indicates a distinct contribution of the FEF to presaccadic modulation in that the FEF is necessary for maintaining the relationship between presaccadic enhancement and stimulus selectivity that is normally observed during the presaccadic period.

\section{Implications for covert attentional modulation}

The modulation of visual activity within area V4 before saccades shares many similarities with that observed during covert spatial attention. For example, both types of modulation involve increased firing rates, reduced trial-to-trial variability, increased stimulus discriminability and shifts in RFs (Tolias et al., 2001; Noudoost et al., 2010; Steinmetz and Moore, 2010). In attention, as in our presaccadic findings, a primarily spatial signal somehow enhances feature selectivity elsewhere in visual cortex (McAdams and Maunsell, 1999). Furthermore, microstimulation of the FEF also increases stimulus selectivity among V4 neurons (Armstrong and Moore, 2007). Although the effect of a spatial signal on stimulus selectivity appears to be counterintuitive, the ability of feature-indifferent spatial feedback to produce enhanced feature selectivity has been reproduced in computation models of attention (Reynolds and Heeger, 2009). In fact, evidence has accumulated over the past several years implicating gaze-related structures in the modulation of visual cortical signals during visual attention (Knudsen, 2007). This evidence is particularly strong for the FEF where studies have demonstrated improvements in psychophysical performance with microstimulation (Moore and Fallah, 2001), as well as improvements in visual signaling in area V4 either with microstimulation (Moore and Armstrong, 2003) or with pharmacological manipulation of FEF neuronal activity (Noudoost and Moore, 2011b). Psychophysical experiments indicate that attention is tied to the target of an impending saccade, with improved discriminability at the sac- cade target relative to other locations (Deubel and Schneider, 1996); we speculate that the decrease in V4 presaccadic selectivity observed following FEF inactivation would disrupt the usual perceptual benefits at the saccade target.

In addition, inactivation of FEF activity leads not only to deficits in saccadic eye movements but also to deficits resembling attentional neglect (Wardak et al., 2006). Results from simultaneous neurophysiological recordings in the FEF and area V4 suggest that the FEF's influence on visual cortical signals during attention involves gamma-band coupling between the areas (Gregoriou et al., 2009). When combined, the above evidence suggests that the FEF's interaction with posterior visual cortex serves to specify the features of visual targets for the accurate guidance of saccades, even when they are planned but not triggered. A recent study provides evidence in support of this notion (Schafer and Moore, 2007). In this view, the presaccadic and covert attentional modulation observed within visual cortex would be consequences of imminent or latent saccade plans, respectively, and would originate in part from the FEF. Consistent with the hypothesis that FEF serves as a source of top-down enhancement of V4 responses, we found that FEF inactivation resulted in greater surround suppression, which is similar to the greater suppression observed when attention is directed to distracters in the surround (Sundberg et al., 2009). Interestingly the increase in surround suppression following the removal of FEF feedback appears to contrast with the effect of inactivating feedback from area V4 to V2 within posterior visual cortex (Nassi et al., 2013). Both effects are consistent with a normalization model of covert attention that accounts for the interaction of top-down modulation with surround influences, particularly in area V4 (Reynolds and Heeger, 2009). Both effects are also consistent with the possibility that the direct excitatory inputs from the FEF to V4 (Anderson et al., 2011) provide a source of gain modulation that may mitigate the divisive normalization due to competing surround stimuli.

Together, the above evidence suggests an important role of the FEF in modulating visual cortical signals both during covert attention and during the preparation of saccades. However, it may be that the FEF's role in both processes does not involve precisely the same neural circuitry. FEF neurons display a mixture of visual, motor, and memory-related properties (Bruce and Goldberg, 1985), and this heterogeneity raises the crucial question of which functional class or classes of neurons mediate the FEF's influence on visual cortical signals. For example, it may be that FEF neurons with motor properties are scarce or absent from the class projecting to visual cortex. This possibility is consistent with a recent study (Gregoriou et al., 2012), and could also help explain the present results in which removal of FEF input did not eliminate presaccadic modulation. Future studies will need to identify the specific class of FEF neurons projecting to visual cortex. Nonetheless, our results indicate that at least area V4's visual activity depends on the FEF more than its saccade-related activity. Whether attentional modulation of V4 activity depends on the FEF, however, remains to be seen. The recent finding that attentional modulation within visual cortex (area MT and MST) persists following inactivation of the SC (Zenon and Krauzlis, 2012) indeed seems to increase the likelihood that the FEF is a source of that modulation. Thus, a loss of covert attentional modulation within V4 following FEF inactivation, combined with the present results, would not only signify a dissociation of the FEF's role in saccadic and attentional modulation but it would further suggest complimentary contributions of the FEF and the SC in those two functions. 


\section{References}

Andersen RA, Asanuma C, Essick G, Siegel RM (1990) Corticocortical connections of anatomically and physiologically defined subdivisions within the inferior parietal lobule. J Comp Neurol 296:65-113. CrossRef Medline

Anderson JC, Kennedy H, Martin KA (2011) Pathways of attention: synaptic relationships of frontal eye field to V4, lateral intraparietal cortex, and area 46 in macaque monkey. J Neurosci 31:10872-10881. CrossRef Medline

Armstrong KM, Moore T (2007) Rapid enhancement of visual cortical response discriminability by microstimulation of the frontal eye field. Proc Natl Acad U S A 104:9499-9504. Medline

Armstrong KM, Chang MH, Moore T (2009) Selection and maintenance of spatial information by frontal eye field neurons. J Neurosci 29:1562115629. CrossRef Medline

Berman RA, Joiner WM, Cavanaugh J, Wurtz RH (2009) Modulation of presaccadic activity in the frontal eye field by the superior colliculus. J Neurophysiol 101:2934-2942. CrossRef Medline

Britten KH, Shadlen MN, Newsome WT, Movshon JA (1992) The analysis of visual motion: a comparison of neuronal and psychophysical performance. J Neurosci 12:4745-4765. Medline

Bruce CJ, Goldberg ME (1985) Primate frontal eye fields: I. Single neurons discharging before saccades. J Neurophysiol 53:603-635. Medline

Buracas GT, Albright TD (2009) Modulation of neuronal responses during covert search for visual feature conjunctions. Proc Natl Acad Sci U S A 106:16853-16858. CrossRef Medline

Chelazzi L, Miller EK, Duncan J, Desimone R (1993) A neural basis for visual search in inferior temporal cortex. Nature 363:345-347. CrossRef Medline

Churchland MM, Yu BM, Cunningham JP, Sugrue LP, Cohen MR, Corrado GS, Newsome WT, Clark AM, Hosseini P, Scott BB, Bradley DC, Smith MA, Kohn A, Movshon JA, Armstrong KM, Moore T, Chang SW, Snyder LH, Lisberger SG, Priebe NJ, et al. (2010) Stimulus onset quenches neural variability: a widespread cortical phenomenon. Nat Neurosci 13:369378. CrossRef Medline

Colby CL, Duhamel JR, Goldberg ME (1996) Visual, presaccadic, and cognitive activation of single neurons in monkey lateral intraparietal area. J Neurophysiol 76:2841-2852. Medline

Desimone R, Schein SJ (1987) Visual properties of neurons in area V4 of the macaque: sensitivity to stimulus form. J Neurophysiol 57:835-868. Medline

Deubel H, Schneider WX (1996) Saccade target selection and object recognition: evidence for a common attentional mechanism. Vis Res 36:18271837. CrossRef Medline

Dias EC, Segraves MA (1999) Muscimol-induced inactivation of monkey frontal eye field: effects on visually and memory-guided saccades. J Neurophysiol 81:2191-2214. Medline

Efron B, Tibshirani RJ (1993) An introduction to the bootstrap. New York: Chapman and Hall.

Fischer B, Boch R (1981a) Selection of visual targets activates prelunate cortical cells in trained rhesus monkey. Exp Brain Res 41:431-433. Medline

Fischer B, Boch R (1981b) Enhanced activation of neurons in prelunate cortex before visually guided saccades of trained rhesus monkeys. Exp Brain Res 44:129-137. Medline

Gattass R, Sousa AP, Gross CG (1988) Visuotopic organization and extent of V3 and V4 of the macaque. J Neurosci 8:1831-1845. Medline

Gregoriou GG, Gotts SJ, Zhou HH, Desimone R (2009) High-frequency, long-range coupling between prefrontal and visual cortex during attention. Science 324:1207-1210. CrossRef Medline

Gregoriou GG, Gotts SJ, Desimone R (2012) Cell-type-specific synchronization of neural activity in FEF with V4 during attention. Neuron 73:581594. CrossRef Medline

Haenny PE, Schiller PH (1988) State dependent activity in monkey visual cortex: I. Single cell activity in V1 and V4 on visual tasks. Exp Brain Res 69:225-244. CrossRef Medline

Hubel DH, Wiesel TN (1968) Receptive fields and functional architecture of monkey striate cortex. J Physiol 195:215-243. Medline

Kaas JH, Huerta MF (1988) Subcortical visual system of primates. In: Comparative primate biology, Vol 4, (Steklis H, ed), pp 327-391. New York: Liss.

Knudsen EI (2007) Fundamental components of attention. Annu Rev Neurosci 30:57-78. CrossRef Medline
Lyon DC, Nassi JJ, Callaway EM (2010) A disynaptic relay from superior colliculus to dorsal stream visual cortex in macaque monkey. Neuron 65:270-279. CrossRef Medline

Mazzoni P, Bracewell RM, Barash S, Andersen RA (1996) Motor intention activity in the macaque's lateral intraparietal area: I. Dissociation of motor plan from sensory memory. J Neurophysiol 76:1439-1456. Medline

McAdams CJ, Maunsell JH (1999) Effects of attention on orientationtuning functions of single neurons in macaque cortical area V4. J Neurosci 19:431-441. Medline

Mohler CW, Wurtz RH (1976) Organization of monkey superior colliculus: intermediate layer cells discharging before eye movements. J Neurophysiol 39:722-744. Medline

Monier C, Chavane F, Baudot P, Graham LJ, Frégnac Y (2003) Orientation and direction selectivity of synaptic inputs in visual cortical neurons: A diversity of combinations produces spike tuning. Neuron 37:663-680. CrossRef Medline

Moore T (1999) Shape representations and visual guidance of saccadic eye movements. Science 285:1914-1917. CrossRef Medline

Moore T, Armstrong KM (2003) Selective gating of visual signals by microstimulation of frontal cortex. Nature 421:370-373. CrossRef Medline

Moore T, Chang MH (2009) Presaccadic discrimination of receptive field stimuli by area V4 neurons. Vision Res 49:1227-1232. CrossRef Medline

Moore T, Fallah M (2001) Control of eye movements and spatial attention. Proc Natl Acad Sci U S A 98:1273-1276. CrossRef Medline

Moore T, Tolias AS, Schiller PH (1998) Visual representations during saccadic eye movements. Proc Natl Acad Sci U S A 95:8981-8984. CrossRef Medline

Nassi JJ, Lomber SG, Born RT (2013) Corticocortical feedback contributes to surround suppression in V1 of the alert primate. J Neurosci 33:85048517. CrossRef Medline

Noudoost B, Moore T (2011a) Control of visual cortical signals by prefrontal dopamine. Nature 474:372-375. CrossRef Medline

Noudoost B, Moore T (2011b) A reliable microinjectrode system for use in behaving monkeys. J Neurosci Methods 194:218-223. CrossRef Medline

Noudoost B, Chang MH, Steinmetz NA, Moore T (2010) Top-down control of visual attention. Curr Opin Neurobiol 20:183-190. CrossRef Medline

Reynolds JH, Heeger DJ (2009) The normalization model of attention. Neuron 61:168-185. CrossRef Medline

Schafer RJ, Moore T (2007) Attention governs action in the primate frontal eye field. Neuron 56:541-551. CrossRef Medline

Schiller PH, True SD, Conway JL (1979) Effects of frontal eye field and superior colliculus ablations on eye movements. Science 206:590-592. CrossRef Medline

Schiller PH, True SD, Conway JL (1980) Deficits in eye movements following frontal eye-field and superior colliculus ablations. J Neurophysiol 44:1175-1189. Medline

Sommer MA, Tehovnik EJ (1997) Reversible inactivation of macaque frontal eye field. Exp Brain Res 116:229-249. CrossRef Medline

Stanton GB, Bruce CJ, Goldberg ME (1995) Topography of projections to posterior cortical areas from the macaque frontal eye fields. J Comp Neurol 353:291-305. CrossRef Medline

Steinmetz NA, Moore T (2010) Changes in the response rate and response variability of area V4 neurons during the preparation of saccadic eye movements. J Neurophysiol 103:1171-1178. CrossRef Medline

Sundberg KA, Mitchell JF, Reynolds JH (2009) Spatial attention modulates center-surround interactions in macaque visual area V4. Neuron 61:952963. CrossRef Medline

Tolias AS, Moore T, Smirnakis SM, Tehovnik EJ, Siapas AG, Schiller PH (2001) Eye movements modulate visual receptive fields of V4 neurons. Neuron 29:757-767. CrossRef Medline

Wardak C, Olivier E, Duhamel JR (2002) Saccadic target selection deficits after lateral intraparietal area inactivation in monkeys. J Neurosci 22: 9877-9884. Medline

Wardak C, Ibos G, Duhamel JR, Olivier E (2006) Contribution of the monkey frontal eye field to covert visual attention. J Neurosci 26:4228-4235. CrossRef Medline

Wurtz RH, Mohler CW (1976) Enhancement of visual responses in monkey striate cortex and frontal eye fields. J Neurophysiol 39:766-772. Medline

Zenon A, Krauzlis RJ (2012) Attention deficits without cortical neuronal deficits. Nature 489:434-437. CrossRef Medline 